\title{
Automated Fault Detection and Diagnostics for Aluminum Threads Using Statistical Computer Vision
}

\author{
Luis Alberto Arróniz Alcántara ${ }^{1}$, Carlos Juárez Toledo², Irma Martínez Carrillo² \\ ${ }^{1}$ Bocar Group, Lerma, Mexico \\ ${ }^{2}$ Professional Academy of UAEMex Tianguistengo, Mexico \\ larroniz03@gmail.com, cjuarezt@uaemex.mx
}

\begin{abstract}
The present work describes the use of statistical computer vision to detect presence-absence of aluminum threads in automotive parts, the vision system is based on a Keyence IV 500 camera and its statistical software. A real case of detection of an industrial aluminum thread was used to demonstrate the effectiveness of the non-invasive machine developed. The results show that using three sequenced tools: brightness adjustment, position reference and area calculation, the repeatability improves by $38 \%$. The study verified the usefulness of the statistical computer vision for fault detection and diagnostics in aluminum threads, a standard statistical analysis of the results presented in the study demonstrates that parts with threads have a punctual performance and, the parts without threads or even without the hole have a statistical normal behavior. The developed method has the ability to automate the correct segregation of good parts with enhanced accuracy avoiding damage to the part, normal in conventional manual methods. The used camera and its brightness compensation demonstrated that environmental light has no effect to the results.
\end{abstract}

Keywords: aluminum threads, fault detection, statistical computer vision, thread inspection, vision inspection.

\section{Introduction}

\subsection{Manufacture of Threads in Aluminum}

Automotive manufacture commonly uses screws to make an assembly between components, the housings of those screws are known as threads. Several normativity exists to define the basic shape of a thread and its manufacturing tolerances.

The basic shape of internal metric threads is described by ISO-68-1:1998 [1]; for aluminum there are two different manufacturing processes that fulfill this. For the first one a previous hole with a diameter equal to the minimum diameter of the thread is made and then a cutter removes material and generates the thread, in the second one a tool deforms the aluminum applying force in the previous hole superior to the elastic limit of the aluminum, the material flows through the male profile and becoming a female laminated thread. In aluminum it's common to use laminated threads for its grater resistance to deformation. 


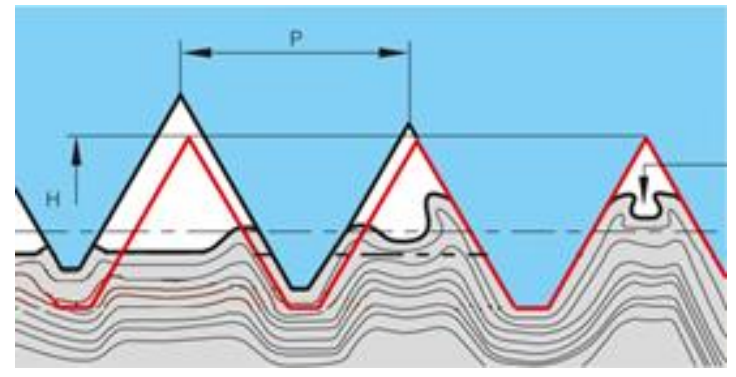

Fig. 1. Representation of laminated threads.

Figure 1, shows a representation of a laminated thread, the red line denotes the basic shape of the thread, the blue zone represents the male tool, the gray zone is aluminum being laminated. Figure 2 shows the real laminated.

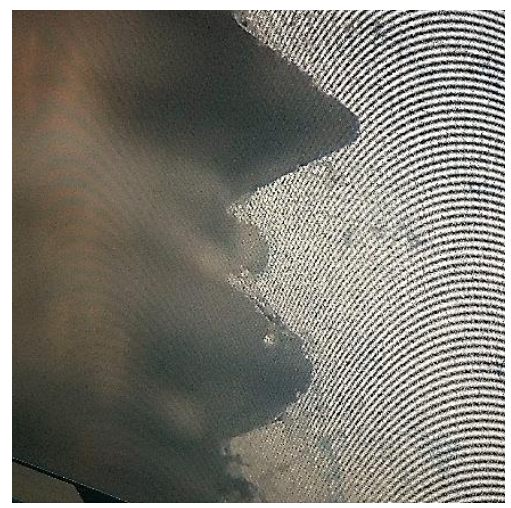

Fig. 2. Laminated thread shape viewed through microscope.

Commonly, laminated threads manufacturing process doesn't have any failure, however if the tool breaks, there is a possibility that the thread doesn't reach the desired depth or not being machined. This failure is what is needed to be detected.

\subsection{Traditional Methods for Thread Inspection}

There are several accepted methods for inspecting threads, however for mass production, go/no-go gauge is the most used. The MSA Manual [2] describes a Gage as a device used to obtain measurement; frequently used to refer to equipment used on production floor; including go and no-go devices. In other words, is an attribute characteristic tool easily used by operators that mechanically compares the interferences between the manufactured part and the device itself.

In the case of threads, gages use pitch diameter to do the inspection, first with the minimum material condition and after with maximum material condition. In the first case, the go gage must be inserted through the whole thread, then the length is measured with a Vernier calibrator, if both comparisons are under tolerance then the no-go gage 
is tried to be inserted but the part must not accept the gage. This inspection method has a cycle time between 12 to 15 seconds for M5 to M12 threads.

The method has three principal disadvantages, cycle time, the probable damage caused in the manufactured part, and the use life of the gage. To solve this problem, the gage is used only for setup analysis and tracking every few hours, however the operator must do a visual inspection to the part, this inspection is called "presence-absence inspection".

\subsection{Discrepant Parts Detection Systems}

The industry is always pushing for suppliers to achieve high volume production with perfect quality rate at low cost. The only way to obtain this objective is to get more efficient process by reducing machine time cycles, and errors in parts and subassemblies.

Presence-Absence detection process have received considerable attention by the Automotive industry, because missing characteristics have associated elevated production and rework cost. Automotive Industry Action Group (AIAG) in FMEA Manual [3] foresees several types of detection systems that can be resumed in the table 1.

Table 1. Detection FMEA resumed table.

\begin{tabular}{lcc}
\hline Criteria & Range & Detection probability \\
\hline $\begin{array}{l}\text { Postprocessing failure detection by visual aids } \\
\text { Postprocessing failure detection by operator }\end{array}$ & 8 & Remote \\
$\begin{array}{l}\text { using variable or attribute gages } \\
\begin{array}{l}\text { Postprocessing failure detection by automated } \\
\text { controls that detects discrepant parts and locks to } \\
\text { prevent posterior postprocessing }\end{array}\end{array}$ & $3-4$ & High \\
\hline
\end{tabular}

According to [3] automated validation machines are placed at the process to ensure that the parts are compliant with the customer's specifications. This validation becomes more important when the customer is Tear 1 or 2, because it doesn't use some of the missing characteristic, and the whole assembly goes to the OEM (Original Part Manufacturer), the cost of the claim increases, and the confidence of the whole productive chain is lost. Those characteristics are called PTC's (Pass Through Characteristics).

Automotive industry normally uses a go/no-go gage to inspect threads, if this verification is made in every part, it could be possible that some of the threads were damage. The gage itself could be affected in its own dimensions, and the part and process could be compromised. The use of gages for $100 \%$ inspection requires an operator dedicated to this verification, the usual time for the job is 12 seconds per characteristic to be verify.

The use of non-invasive media to detect presence-absence of threads has many advantages (see Table 2), however when a Repeatability-Reproducibility study is 
performed, the non-invasive media has a low performance, therefore this kind of inspection methodologies are avoided.

Table 2. Comparison between gages and non-invasive media.

\begin{tabular}{lll}
\hline Criteria & Use of gage go No-go & Use of non-invasive media \\
\hline Cycle Time & 12 seconds minimum & 0.5 seconds maximum \\
Operators & Dedicated & Shared \\
Damages & Highly possible & Non-Possible \\
Repeatability & High & Low \\
Reproducibility & High & Low \\
\hline
\end{tabular}

No invasive methods could be used to support the diagnosis of production, assuring the characteristic without damaging the part. The use of statistical methods to determine when a machine is trustworthy enough to detect not conforming product and guaranty the compliance with the customer regulations. The main porpoise of this paper is to present a visual machine that detects the presence-absence of CNC laminated threads with a high performance in repeatability and reproducibility, demonstrating the viability of the method.

\section{Related Work}

The main works for the detection of faults are enlisted below.

In 1982 Baumann [4] documented the first production implementation of the General Motors "Consight Vision System", foundry successfully sorted up to six different castings at up to 1,400 an hour from a belt conveyor us-ing three industrial robots in a harsh manufacturing environment.

Vedang [5] fully describe at least ten documented works dated between 1988 and 2014, he himself presented in 2015 a Comparative study of machine vision-based methods for fault detection in an automated assembly machine [6], that compares three different methods, Gaussian Mixed Models and Blob Analysis, optical flow method and running average method.

Gonzalez [7] describes in 2013 a vision-based system presence-absence inspection of automotive subassemblies, using artificial vision for identifying the correct position of holes, threads and welds points using a laser pro-filometer and stroboscopic light.

Urrea [8] in 2004 uses the Hough transform for detecting lines in a low-level vision system, he uses this algorithm in a toy mobile.

Cortes [9] presented in 2010 a computer vision system for quality control in production, in his paper he describes the use of Matlab for digital processing for RGB and CMYK decomposition.

In 2013 Londoño [10] generated an introduction to artificial vision through laboratory guides using Matlab.

Vedang [5] in 2014, presented the Effect of illumination techniques on machine vision inspection for automated assembly machines. 
Braggins [11] provides the actual illumination features for machine vision.

There are many processes that uses vision systems to detect faults or follow tracks, in example, in 2007 Min-Goo Kang [12] presented an automatic weld seam tracking method using laser vision, in 2017 Sun Huaiyuan [13] used a vision based machine to assure the correct package of pharmaceutical drugs, in 2016 Ji Yeon Lee [14] describes a method to detect faults in the package of electronic chips.

The actual work presents a machine that detects the presence-absence of CNC formed threads, based in Keyence IV cameras and Keyence contrast detectors. The next section describes the camera and all the involved configurations.

\section{Machine for PTC's Inspection}

\subsection{Machine Design}

The main parts of the machine are shown in Fig. 3, it consists of: one nest where the part is clamped and inspected, six cameras that verify the presence-absence of several product specification characteristics and a pneumatic cylinder that marks the conditions piece.

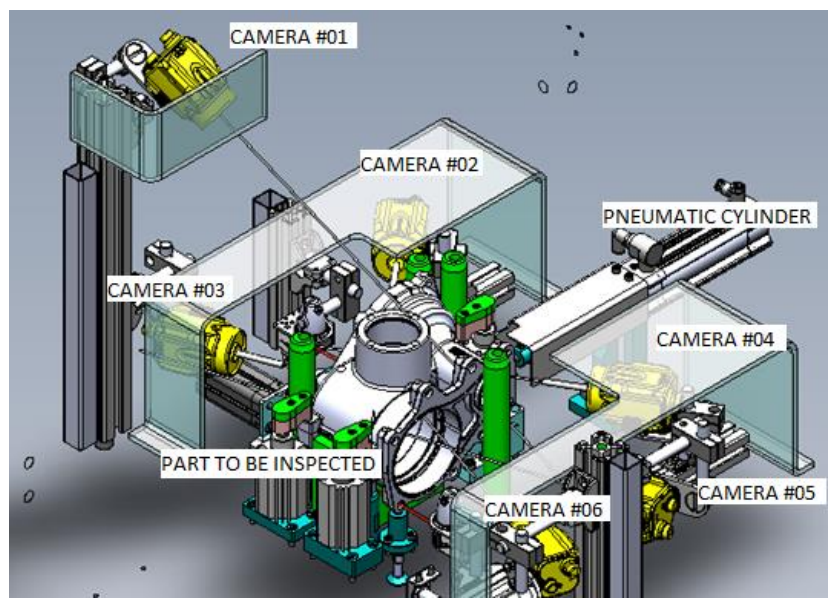

Fig. 3. Automated Vision Base PTC detection machine.

The machine uses a Siemens PLC to control all the functions, the human machine interface (HMI) helps to communicate failures and to accept reestablishment commands, opaque polycarbonate was installed to reduce environmental lighting to minimum, the inspection machine rate is 170 parts per hour. Siemens PLC and HMI are programed according the process, Keyence IV cameras were setup to fulfill three primary requirements, best focus, sufficient bright and the best contrast between an ok and nok part.

During the automatic cycle cameras are operated simultaneously, each one sends an ok or nok signal to the PLC and if the trial of all sensor is ok, led lights are turned on 
at a visual aid (see fig 4) in this case the part is unclamped otherwise, the correspondent led blinks showing which characteristic is not accepted, the sequence is interrupted until the supervisor recognize the fault.

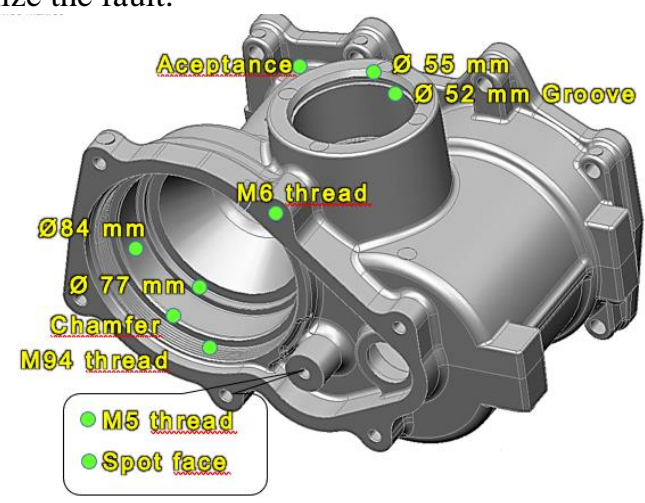

Fig. 4. Visual Aid.

The system design includes steps such as selection, location and adjustment of cameras, environmental light controls, load-unload nest design, clamping method, PLC-HMI program. The design accomplishes the next norms NOM-004-STPS-1999, ISO 12100, ISO TR 14121-2, ISO 1420, ISO 4413, IEC 60204-1, IEC 61140 and IEC 62061.

A critical task to implement the project is the calibration of the cameras, the present work will detail the configuration of the cameras to inspect a roll tap M5 thread in aluminum automotive part with a spot face in fig 5 .

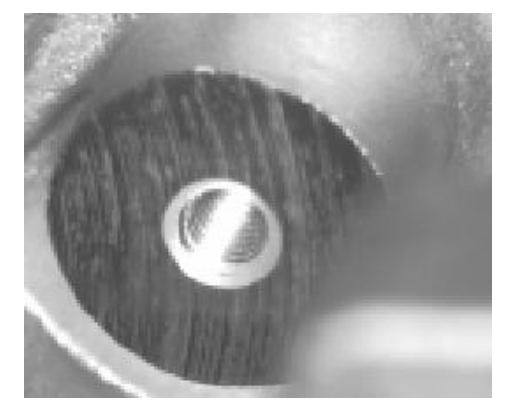

Fig. 5. M5 thread with spot face.

\subsection{Camera Configuration for Thread Detection}

Cameras have been used to detect the presence of threads, though, it has been proven that the variation in the illumination and the contrast of the image can cause measurement errors.

Keyence IV cameras have an autofocus system however, it could be set in different positions between $0 \mathrm{~mm}$ and $500 \mathrm{~mm}$, brightness adjustment could be set between 1 (dark) and 120 (bright) and it has three different imaging modes: 
- Normal less noise.

- $\quad$ HDR special adjustment for metals.

- High gain short exposure time but low quality of the image.

The parameters for M5 thread capture image were set as follows: Bright 71 with an exposure time of $0.36 \mathrm{~ms}$ and HDR mode is selected, focus selected was $110 \mathrm{~mm}$, and a magnification area of $2 \mathrm{x}$ with edge emphasis turned on. This increments the outline recognition. The image of figure 6 was set as master image and a compensation of brightness was set.

Three hundred images were captured to be used as samples of the production line, those images were compared with the master image in Keyence IV configured with an area tool. Figures 7 a) and 7 b) show the results using the compensation of brightness.

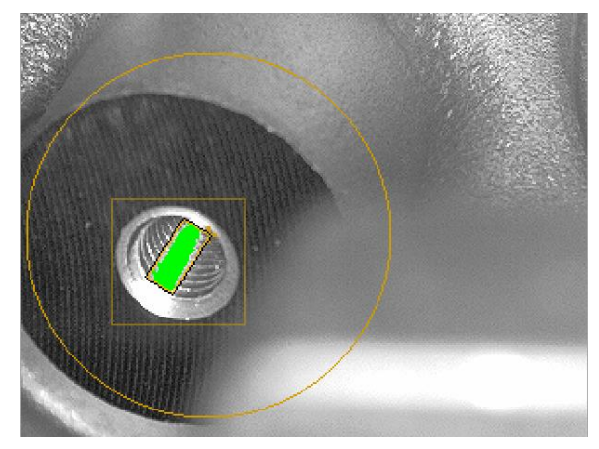

Fig. 6. Common configuration for thread detection.

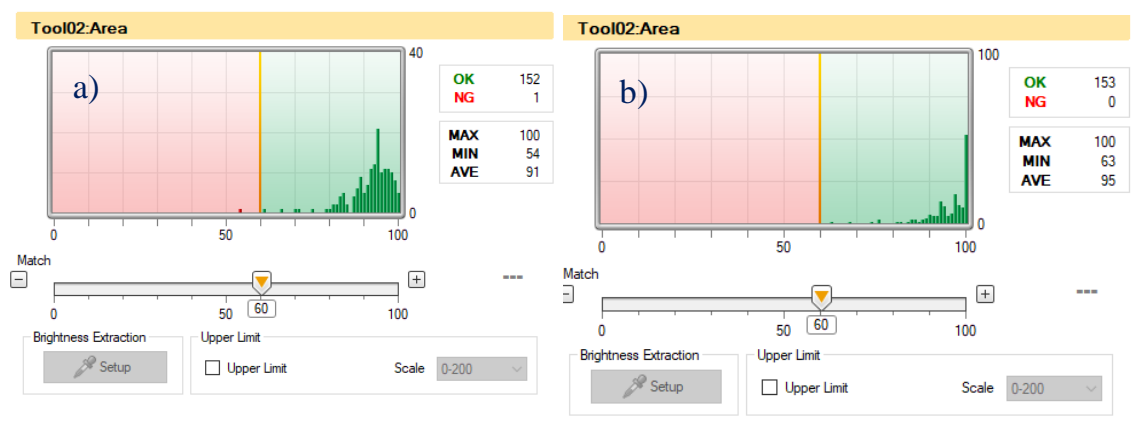

Fig. 7. a) Statistical results without brightness compensation, and b) Statistical result with bright compensation.

Two tools were used to configure the camera:

-Position tool, it looks for edges in the part that are located always at the same distance to the area to be analyzed, it is important to locate edges in axis $\mathrm{x}$ and $\mathrm{y}$, fig 8 shows the configuration of the position tool. Green patterns are the edges to be validated, yellow patterns are erased from the images to avoid noise. As seen, an arc and a circle are validated, the result is that all the next tools must be referred to the position tool. 


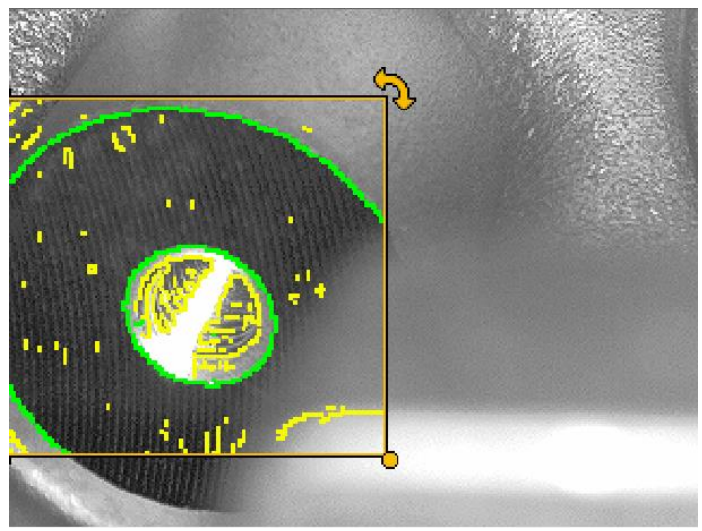

Fig. 8. Position tool adjustment.

-Area tool, the area tool is used to detect high bright in the selected area, the tool was configured to detect thread pitches avoiding the center bright. Blue square in fig 9 is the bright compensation for the analysis, green pattern are the thread pitches, a mask in the center allows the camera to ignore the high bright area that is coincident with holes without threads.

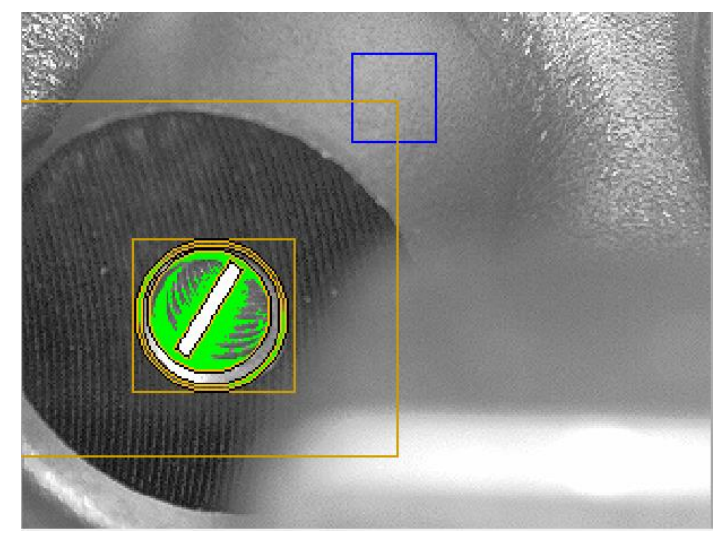

Fig. 9. Thread detection area tool configuration.

The inspection was made in less than a second, as the machine has 6 cameras and several sensors installed, the total cycle time is 20 seconds including load and unload.

\section{$4 \quad$ Results and Discussion}

The performance of the adjustment was tested on 300 samples out of which the 185 data sets were for normal operation sequence of the machine (CNC formed thread M5), 75 were for parts that only include a hole nor a thread, and 40 were for parts that has no hole. To evaluate the performances, the results obtained were analyzed with the 
statistical tool included in the Keyence IV sensor simulation software. Fig. 10 shows the behavior, Green data are good parts, red data are for not good parts, as seen there are 2 sections in red that correspond to no presence of thread and no presence of hole.

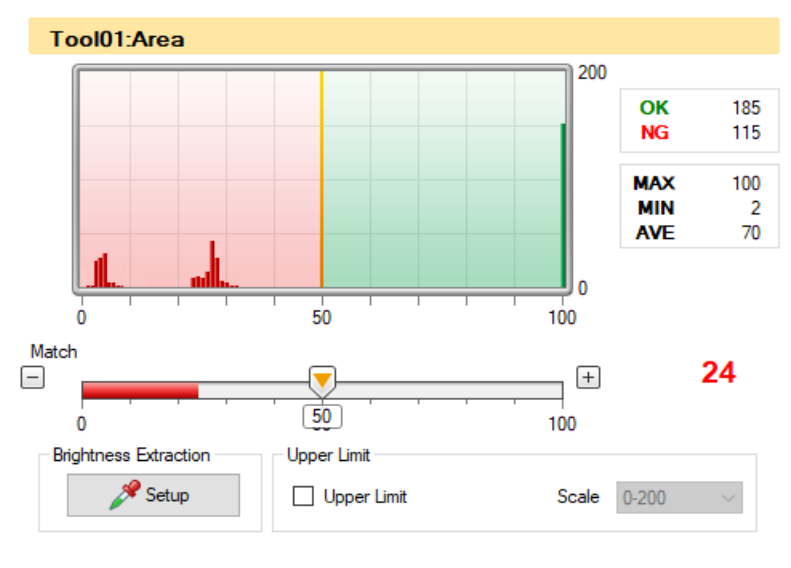

Fig. 10. Equipment performance using statistical results.

Even when the results of good parts have no statistical normal behavior, the difference between the good parts and the not good parts is 78 units of 100 , that significantly contributed to increase the confidence on the detection of the absence of threads, Hence the led included in the visual aid helps the operator and the supervisor to protect the customer.

\section{Conclusions}

The effectiveness of the developed non-invasive machine using the statistical computer vision is presented, the article shows that a correct configuration of the camera improves the performance of the machine. Thread parametrization requires special training for process engineers, however operators have a confident tool to segregate ok and nok parts. It is concluded that presence-absence of threads with automated visual controls will assure the protection of the customer and complies the demand of AIAG.

The time difference between, 20 seconds inspecting 15 characteristics, with 12 seconds only for verify the M5 thread with the use of a gage demonstrated that automated fault detection machines are faster and effective to isolate good parts.

Plans for future work include parametrization in different internal threads between M5 to M94 using the actual work as a base, the parameters would be tested on their ability to detect faults.

\section{References}

1. ISO: ISO-68-1:1998. ISO general purpose screw Threads-Basic profile. 
2. Automotive Industry Action Group, MSA MANUAL REV 04, AIAG (2010)

3. Automotive Industry Action Group, FMEA MANUAL REV 04, AIAG (2010)

4. Baumann, R., Wilmshurst, D.: Vision System Sorts Castings at General Motors Canada. Emerald, pp. 145-150 (1982)

5. Vedang, C., Surgenor, F.: Effect of Illumination Techniques on Machine Vision Inspection for Automaed Assembly Machines. In: Proceedings of The Canadian Society for Mechanical Engineering International Congress, pp. 1-6. Toronto, Ontario, Canada (2014)

6. Chauhan, V., Surgenor, B.: A comparative Study of Machine Vision Based Methods for Fault Detection in an Automated Assembly Machine. In: 43rd Proceedings of the North American Manufacturing Research, pp. 416-428 (2015)

7. González, A., Ramírez, A., Padilla, J.A., Morales, R.: Sistema basado en visión para inspección del tipo ausencia/presencia de subensambles automotrices. Científica 17(1), pp. 29-37 (2013)

8. Urrea, J. P., Ospina, E.: Implementación de la Transformada de Hough para la Detección de Líneas para un Sistema de Visión de Bajo Nivel. Scientia Et Technica X(24), pp. 79-84 (2004)

9. Cortés, J.A., Medina, F.A., Mendoza, J.A.: Computer Vision System for Quality Control in Production, Scientia Et Technica XVI(45), pp. 130-134 (2010)

10. Londoño, V., Marín, J., Arango, E.: Introducción a la Visión Artificial Mediante Prácticas de Laboratorio Diseñadas en Matlab. Tecno Lógicas, pp. 591-603 (2013)

11. Braggins, D.: Illumination for Machine Vision. Sensor Review, 20(1), pp. 20-23 (2000)

12. Kang, M.: Laser vision system for automatic seam tracking of stainless steel pipe welding machine. In: International Conference on Control, Automation and Systems, pp. 1045-1051. Seoul, South Korea (2007)

13. Huaiyuan, S.: The Detection System for Pharmaceutical Bottle-packaging Constructed by Machine Vision Technology. In: Third International Conference on Intelligent System Design and Engineering Applications, pp. 1423-1425. Hong Kong, China (2013)

14. Lee, J. Y.: Development of vision system for defect inspection of electric parts in the tape and reel package. In: 16th International Conference on Control, Automation and Systems (ICCAS), pp. 437-439. Gyeongju, South Korea (2016) 